\title{
Bolivia debe PrEPararse ante las nuevas infecciones del VIH
}

Como publican datos estadísticos del Programa Nacional ITSVIH/sida del Ministerio de Salud y del SEDES Cochabamba, en el 2017 se determinó que los casos de infecciones por VIH en Bolivia incrementaron en un 30\%, comparado con la gestión 2016 y en algunas regiones rurales de Cochabamba aumentaron entre el 100 al 300\% ${ }^{1}$.

Existe aún un diagnóstico tardío para $65 \% \%^{2}$, de personas que llegan a los servicios hospitalarios de Cochabamba en etapa de sida, muchas de estas personas continúan falleciendo.

Según información del Ministerio de Salud, el VIH/Sida tiene una prevalencia en la población general de $0,16 \%$ y $0,80 \%$ en gestantes; en trabajadoras sexuales $0,57 \%^{3}$ (3). No obstante, esta cifra asciende en la población GLBT-HSH (Gey, Lesbiana, Bisexual, Transexual-Hombres que tienen Sexo con Hombres) a 11,6\% y en la población Trans a 19,70\% (proyecciones EPPSpectrum) $)^{4}$.

\section{LA NUEVA ESTRATEGIA} MUNDIAL DE PREVENCIÓN COMBINADA PrEP (prevención ante exposición)

La PrEP es una estrategia de prevención dirigida a personas que no están infectadas con el VIH, que tienen comportamientos sexuales con alto riesgo de transmisión y que, por alguna $\mathrm{u}$ otra razón, no utilizan preservativos $\mathrm{u}$ otros medios de prevención.

La PrEP consiste en tomar un medicamento (Truvada $\AA$ ) antes de una exposición a una relación sexual de alto riesgo, para reducir las probabilidades de infectarse. Es una combinación de dos medicamentos (Tenofovir y Emtricitabina), utilizados en el tratamiento para el VIH. Los estudios han mostrado que la PrEP es altamente eficaz para prevenir el VIH, si se usa tal como se prescribe.

La PrEP implica realizar controles médicos regularmente, porque puede provocar algunos efectos secundarios menores, como náuseas, que generalmente se atenúan con el tiempo.

Las recomendaciones de la OMS para autorizar del uso de la PrEP son sustentados por las principales agencias regulatorias de medicamentos (la FDA en Estados Unidos de América) ${ }^{5}$. La PrEP responde a evidencias científicas sobre su eficacia producida por estudio clínicos y comportamentales de los últimos cinco años.

Los estudios han mostrado que la PrEP reduce en más del $\mathbf{9 0 \%}$ el riesgo de contraer el VIH a través de las relaciones sexuales cuando se toman los medicamentos de acuerdo a las indicaciones del médico. Entre los usuarios de drogas intravenosas, la PrEP reduce el riego de contraer el VIH en más del $70 \%$ cuando se toman los medicamentos, según las indicaciones del médico ${ }^{6}$

Es importante informar sobre la PrEP y ver las posibilidades para implementarla en Bolivia (ventajas, costos, dificultades, etc.). La PrEP ya se usa en varios países de la región, pero solo acceden a esta quienes pueden pagarla. Sin embargo, la OMS ha precalificado genéricos de la PrEP con un costo 33 veces menor a el producto original $^{7}$.

De manera indirecta, el uso de la PrEP aumenta el número de personas que se realizan la prueba de VIH para saber si tienen o no el VIH antes de iniciar la prevención.

Si más personas son diagnosticadas oportunamente y tratadas inmediatamente (test and treat), la evolución de su salud será mejor y disminuirá la capacidad de transmitir el VIH en las relaciones sexuales no protegidas. Con estas medidas, se cumple otra de las nuevas estrategias de prevención que es el Tratamiento como Prevención (TasP), dirigido a las personas que tienen el diagnóstico de VIH.

Uno de los retos que plantea la discusión es la falta de información sobre la PrEP en Bolivia. Es difícil organizar un debate cuando los médicos, las poblaciones o personas más expuestas al VIH carecen de la información básica sobre el tema y cuando las autoridades nacionales del Ministerio de Salud no crean espacios para abordar la temática.
Las personas que no están infectados con el VIH tienen el derecho a acceder a los avances de la ciencia, que permitan seguir siendo VIH negativos.

Exhortamos a que nuestras autoridades del Ministerio de Salud, se informen sobre la PrEP, su importancia en la lucha contra el VIH, creando espacios de información y discusión sobre las nuevas estrategias, que lamentablemente no las mencionan en los programas oficiales. $\mathrm{Su}$ responsabilidad no debe limitarse a proveer algunos condones (más económicos en teoría).

Es importante tomar las siguientes consideraciones científicas, epidemiológicas, culturales y éticas, sobre el VIH.

Actualmente sabemos que:

-Existe un medicamento eficaz que reduce la transmisión del VIH en más del $90 \%$.

-La epidemia del VIH en Bolivia está concentrada en las poblaciones trans y $\mathrm{HsH}$, según los datos del Ministerio de Salud.

-Las personas más expuestas al riesgo no utilizan el preservativo, por alguna razón.

-Este medicamento es una opción, para ellas, de protegerse de la transmisión del VIH.

-Con el esfuerzo de todos los países, se podría terminar la epidemia (recomendaciones internacionales).

Brindar la información sobre la PrEP a la población, constituye el cumplimiento del derecho a la información, tal como lo indica la ley 3729 , articulo $18^{8}$.

Las personas más expuestas al VIH, deben recibir información ahora sobre la PrEP, mañana será demasiado tarde. Las autoridades del gobierno central, departamental y los municipios tienen la responsabilidad directa o indirecta que la transmisión del VIH continúe aumentando o disminuya.

El uso es voluntario, la PrEP, es como los anticonceptivos, nadie está obligado a utilizarlos. El uso de la PrEP debe ser una decisión personal e informada.

La sexualidad está llena de prejuicios, 
estereotipos, pudor y cuestiones morales. Las personas habitualmente no hablan con sus médicos, ni con sus amigos más próximos sobre la no utilización del condón. La PrEP permite que las personas accedan a una mayor protección.

De la misma manera que la sociedad civil luchó por el acceso al tratamiento de las personas con $\mathrm{VIH}$, hoy es necesario realizar acciones para aumentar el acceso a la PrEP.

No se trata de sacar recursos de los presupuestos de tratamiento del VIH para dirigirlos a la prevención, o viceversa, sino de tener programas de prevención, tratamiento y cuidado de acceso universal basado en las evidencias científicas y con los recursos presupuestarios necesarios.

La PrEP es una herramienta más para el control y erradicación de la epidemia.

\section{AUTOR:}

Dr. Edgar Valdez Carrizo

Director del Instituto para el Desarrollo Humano IDH- Bolivia

Especialización en Salud Pública, investigación y desarrollo.
REFERENCIAS

1. Zapana V. Los casos de VIH en Bolivia se incrementaron en un $\mathbf{3 0} \%$. Página Siete. 2017 May. Disponible en: http://www.paginasiete. bo/sociedad/2017/5/1/casos-boliviaincrementaron-136149. html

2. $R$ Quiroga. Factores que influyen en el retraso del diagnóstico del VIH. Gac. Med. Bol. 2016 Dec; 39(2). Disponible en: http://www.scielo.org. bo/scielo.php?script=sci_arttext\&pid =S1012-29662016000200003

3. Valdez E. PrEPararse para enfrentar al VIH. Los Tiempos. 2018 Jan 31. Disponible en: http://www.lostiempos. com/actualidad/opinion/20180131/ columna/prepararse-enfrentar-al-vih

4. POLICY O. Espectrum Instructivo. 2005 Mar: p. 1-17. Disponible en: http:// data.unaids.org/topics/epidemiology/ manuals/spectrum_quickstart_05_ es.pdf.

5. FDA. TRUVADA (emtricitabine/ tenofovir disoproxil fumarate)-FDA. 2014 Jun 18: 1-34. Disponible en: https:// www.accessdata.fda.gov/drugsatfda_ docs/label/2005/21752s002/bl.pdf

6. infoSIDA. [Online]; 2017. Available from:https://infosida. nih.gov/understanding-hiv-aids/ fact-sheets $/ 20 / 85 /$ profilaxis preexposicion-prep-

7. Baruch $R$ A. PrEP en América Latina: Información sobre Profilaxis Pre-Exposición para Hombres que Tienen Sexo con Hombres. researchgate.net. 2016 Jun. Disponible en: https://www.researchgate.net/ publication/305721801_PrEP_en_ America_Latina_Informacion_sobre_ Profilaxis_Pre-Exposicion_para_ Hombres_que_tienen_Sexo_con_ Hombres/references

8. Bolivia Ministerio de Salud [Online]; 2007. Disponible en: https:// www.minsalud.gob.bolimages/ Documentacion/normativa/LeyN_3729. $p d f$ 